\title{
Correction to: Antifungal activity of Myrtus communis against Malassezia sp. isolated from the skin of patients with pityriasis versicolor
}

\author{
Aleksandra Barac ${ }^{1,2} \cdot$ Matthew Donadu $^{3}$. Donatella Usai ${ }^{3}$. Vesna Tomic Spiric ${ }^{1,4}$ - Vittorio Mazzarello ${ }^{3}$. \\ Stefania Zanetti ${ }^{3} \cdot$ Ema Aleksic $^{5} \cdot$ Goran Stevanovic $^{1,2} \cdot$ Natasa Nikolic $^{2} \cdot$ Salvatore Rubino $^{3}$
}

Published online: 2 January 2018

(c) Springer-Verlag GmbH Germany, part of Springer Nature 2017

\section{Correction to: Infection \\ https://doi.org/10.1007/s15010-017-1102-4}

The original version of this article unfortunately contained two mistakes in authors' names.

The correct names of the seventh and ninth authors are Ema Aleksic and Natasa Nikolic, respectively.

The original article has been corrected.

The original article can be found online at https://doi.org/10.1007/ s15010-017-1102-4.

Vittorio Mazzarello

abarac@jidc.org

1 Faculty of Medicine, University of Belgrade, Dr Subotica 1, 11000 Belgrade, Serbia

2 Clinic for Infectious and Tropical Diseases, Clinical Centre of Serbia, Bulevar Oslobodjenja 16, 11000 Belgrade, Serbia

3 Department of Biomedical Sciences, University of Sassari, V. le San Pietro 43/C, 07100 Sassari, Italy

4 Clinic for Allergology and Immunology, Clinical Centre of Serbia, Dr Koste Todorovica 2, 11000 Belgrade, Serbia

5 Faculty of Stomatology, University of Business Academy, Zarka Zrenjanina 179, 26000 Pancevo, Serbia 